\title{
Robust Zonotopic Observer Design: Interval Observer versus Set-membership Approaches
}

\author{
Masoud Pourasghar, Vicenç Puig and Carlos Ocampo-Martinez
}

\begin{abstract}
The aim of this paper is to provide a robust observer design using a Linear Matrix Inequality (LMI) technique along with the analysis and comparison of the interval observer and set-membership approaches within the state estimation framework. The propagation of the uncertainty is taken into account considering a zonotopic-set representation. Both approaches are compared and related using Linear Quadratic Regulator (LQR). Finally, a simulation example based on a three-tank system is employed to both illustrate and discuss the effectiveness of the proposed approach.
\end{abstract}

\section{INTRODUCTION}

Model-based approaches is nowadays a well-established approach that is becoming increasingly important in the field of automatic control [1]. Those approaches rely on the use of a mathematical model describing the system behavior [2]. However, due to the effect of model uncertainty, unknown disturbances and noises, the mismatch between the actual and estimated process behaviours is non-negligible even if there are no process faults [3]. Thus, dealing with the uncertainties is an important issue, and plays a key role in model-based approaches [4], [5].

Moreover, the state estimation problem is an important topic in control theory since knowing the state of a system might be crucial for implementing many control and fault diagnosis methods. Based on the literature, there are several approaches for estimating the system states (and outputs) depending on the way that uncertainties are modeled. In recent years, several methods have been developed and introduced to explicitly consider such uncertainties in the models [6]. See [7], [8] and [9] for more information related to different available ways for modeling the effect of uncertainties in model-based approaches. In particular, there exist two different paradigms for considering the uncertainty in the model. In the stochastic approaches, uncertainties are represented using random variables, while in the deterministic approaches, uncertainties are assumed unknown but bounded by means of different type of sets, e.g., interval boxes, polytopes, ellipsoids and zonotopes [6], [8], [3]. According to [10], polytopes provide tighter enclosures than interval boxes. However, the main drawback of using general polytopes is related to the complexity of vertices enumeration with respect to the space dimension. But, using zonotopes, basic set operations can be reduced to simple matrix calculations, fact that has recently motivated the

Automatic Control Department, Universitat Politècnica de Catalunya, Institut de Robòtica i Informàtica Industrial (CSIC-UPC), Supervision, Safety and Automatic Control Research Center (CS2AC), C/. Llorens i Artigas 4-6, 08028 Barcelona, Spain \{mpourasgharlaf, vpuig, cocampo\}eiri.upc. edu use of zonotopes for modeling the effect of uncertainties [11]. Within the family of deterministic approaches, interval observer approach (IOA) and set-membership approach (SMA) have been introduced, separately. The state estimation provided by both approaches is given in a form of a set of states at each time instant [12], [13]. IOA allows to estimate the state set one time instant ahead based on the set estimated in the previous time instant [14]. On the other hand, the SMA-based state estimation is an alternative approach for estimating the state of the system including the measured output and bounded noises. SMA allows to estimate the state set by means of the intersection between the predicted state set in the previous time instant with the strip obtained by using the measurement [4].

The main contribution of this paper is to establish the mathematical comparison of IOA and SMA into the Linear Matrix Inequality (LMI) framework establishing the condition that both approaches can obtain the identical results. With this aim, the zonotopic representation of a set is considered for propagating the effect of uncertainties due to its simple matrix calculation in comparison with the huge number of vertices of the equivalent polytopes. Finally, a well-known benchmark based on the three-tank system is used as a case study for illustrating the results presented in the paper.

The structure of the paper is the following: The problem formulation is presented in Section II. The state estimation principle for each approach is discussed in Section III. The way to relate the IOA and SMA is discussed in Section IV. In Section V, a three-tank system is used to illustrate the results obtained in the paper. Finally, conclusions are drawn in Section VI.

\section{Notation}

Throughout this paper, $\mathbb{R}^{n}$ denotes the set of $n$ dimensional real numbers and $\oplus$ denotes the Minkowski sum. The matrices are written using capital letter, e.g., $A$ and the calligraphic notation is used for denoting sets, e.g., $\mathcal{X}$

\section{PRoblem Formulation}

\section{A. Problem set-up}

The class of systems considered in this paper is that of a discrete-time invariant linear uncertain system with the following state-space form:

$$
\begin{aligned}
x_{k+1} & =A x_{k}+B u_{k}+E_{\omega} \omega_{k}, \\
y_{k} & =C x_{k}+E_{v} v_{k},
\end{aligned}
$$


where $u \in \mathbb{R}^{n_{u}}, y \in \mathbb{R}^{n_{y}}$ and $x \in \mathbb{R}^{n_{x}}$ are the input, the output and the state vectors, respectively. Moreover, $A \in$ $\mathbb{R}^{n_{x} \times n_{x}}, B \in \mathbb{R}^{n_{x} \times n_{u}}$ and $C \in \mathbb{R}^{n_{y} \times n_{x}}$ are the state-space matrices. Both state disturbance and process noise vectors are defined by $\omega \in \mathbb{R}^{n_{x}}$ and $v \in \mathbb{R}^{n_{y}}$, respectively. Moreover, $E_{\omega}$ and $E_{v}$ are the distribution matrices with appropriate dimensions and $k \in \mathbb{N}$ denotes the discrete time.

Moreover, the measurement noise and process disturbances are assumed to be unknown but bounded, i.e.,

$$
\begin{aligned}
\mathcal{W} & =\left\{\omega_{k} \in \mathbb{R}^{n_{x}}:\left|\omega_{k}-c_{\omega}\right| \leq \bar{\omega}, c_{\omega} \in \mathbb{R}^{n_{x}}, \bar{\omega} \in \mathbb{R}^{n_{x}}\right\}, \\
\mathcal{V} & =\left\{v_{k} \in \mathbb{R}^{n_{y}}:\left|v_{k}-c_{v}\right| \leq \bar{v}, c_{v} \in \mathbb{R}^{n_{y}}, \bar{v} \in \mathbb{R}^{n_{y}}\right\},
\end{aligned}
$$

where $c_{\omega}, \bar{\omega}, c_{v}$ and $\bar{v}$ are constant vectors. Notice that the inequalities associated to $\left|\omega_{k}-c_{\omega}\right|$ and $\left|v_{k}-c_{v}\right|$ in (2) are considered component-wise.

Since the paper considers a zonotopic representation of state estimation stet, (2) can be rewritten using a zonotopic representation as follows:

$$
\begin{gathered}
\mathcal{W}=\left\langle c_{\omega}, R_{\omega}\right\rangle, \\
\mathcal{V}=\left\langle c_{v}, R_{v}\right\rangle,
\end{gathered}
$$

where $c_{\omega}$ and $c_{v}$ denote the centers of the disturbance and noise bouding zonotopes, respectively, with their generator matrices $R_{\omega} \in \mathbb{R}^{n_{x} \times n_{x}}$ and $R_{v} \in \mathbb{R}^{n_{y} \times n_{y}}$, respectively.

Assumption 2.1: The pair $\{A, C\}$ of the dynamical model (1) is detectable.

Assumption 2.2: Disturbance and noise bounds represented in (3) are assumed to be bounded by the unitary hypercube zonotope centered at the origin, i.e., $\forall k \geq$ $0, \omega=\in[-1,1]^{n_{\omega}}=\left\langle 0, I_{n_{\omega}}\right\rangle$ and $v \in[-1,1]^{n_{v}}=$ $\left\langle 0, I_{n_{v}}\right\rangle$ where $I_{n_{\omega}} \in \mathbb{R}^{n_{\omega} \times n_{\omega}}$ and $I_{n_{v}} \in \mathbb{R}^{n_{v} \times n_{v}}$ denote the identity matrix.

Henceforth, the index $k+1$ will be replaced by + and $k$ will be omitted for the sake of simplified notations. Then, the dynamical model (1) is rewritten as

$$
\begin{aligned}
x_{+} & =A x+B u+E_{\omega} \omega, \\
y & =C x+E_{v} v .
\end{aligned}
$$

Consequently, the index $k-1$ will be replaced by - when it will be needed throughout the paper.

\section{STATE ESTIMATION PRINCIPLES}

\section{A. Zonotopic IOA}

Monitoring the dynamical model (4) can be done by designing a Luenberger observer of the form

$$
\hat{x}_{+}=A \hat{x}+B u+L(y-\hat{y}),
$$

where $\hat{x} \in \mathbb{R}^{n_{x}}$ is the state estimation. Moreover, the observer gain $L \in \mathbb{R}^{n_{x} \times n_{y}}$ should be computed such that $(A-L C)$ is a Schur matrix (i.e., all eigenvalues with a module less than unity) that guarantees the convergence of the observer.

Assumption 3.1: The initial state $x_{0}$ belongs to the set $\mathcal{X}_{0}^{i o}=\left\langle c_{x, 0}^{i o}, R_{x, 0}^{i o}\right\rangle$, where $c_{x, 0}^{i o} \in \mathbb{R}^{n_{x}}$ denotes the center and $R_{x, 0}^{i o} \in \mathbb{R}^{n_{x} \times n_{R_{x, 0}^{i o}}}$ is a non-empty matrix containing the generators matrix of the initial zonotope $\mathcal{X}_{0}^{i o}$.
Therefore, according to [15], the resulting interval observation of the dynamical model (4) can be obtained by using Proposition 3.1.

Proposition 3.1: Considering the dynamical model (4) and the Luenberger observer structure in (5), the zonotope center and shape matrix of the state-bounding observer, i.e., $\hat{\mathcal{X}}^{i o}=\left\langle c_{x}^{i o}, R_{x}^{i o}\right\rangle$, can be computed using

$$
\begin{aligned}
& c_{x}^{i o}=c_{p}^{i o}+L^{i o}\left(y_{-}-C c_{p}^{i o}\right), \\
& R_{x}^{i o}=\left[\left(I-L^{i o} C\right) \bar{R}_{p}^{i o}-L^{i o} E_{v}\right] \text {, }
\end{aligned}
$$

where

$$
\begin{aligned}
c_{p}^{i o} & =A c_{x,-}^{i o}+B u_{-}, \\
R_{p}^{i o} & =\left[\begin{array}{ll}
A \bar{R}_{x,-}^{i o} & E_{\omega}
\end{array}\right] .
\end{aligned}
$$

Proof: According to [16], the computation of the state-bounding observer using zonotopes and the Luenberger observer structure in (5) for the dynamical model (4) can be divided into two steps: prediction step and update step. In the prediction step, the estimated set at time instant $k-1$ is used to compute a priori state estimation. Then, this state estimation is updated with the information from the measurement (the obtained estimated set in the update step is called a posteriori estimated set). Therefore, a priori state estimation set $\mathcal{P}^{i o}=\left\langle c_{p}^{i o}, R_{p}^{i o}\right\rangle$ is computed using the system matrices $A$ and $B$, and the input at the previous time step, i.e., $u_{-}$, as in (7). Then, the obtained set can be updated using the observer gain $L^{i o}$ as in (6).

The observer gain $L^{i o}$ provides degrees of freedom to tune the system monitoring with respect to its aim, i.e., with the goal of optimizing the state-bounding observer according to some given criterion. An optimal tuning of the observer gain matrix $L^{i o}$ based on a set-based optimization criterion expressing the desired state estimation performance is addressed next.

\section{B. Zonotopic SMA}

Alternatively, one possible way for computing the statebounding zonotope is to make use of the SMA according to [4] and Proposition 3.2.

Proposition 3.2: Considering the dynamical model (4), the center $c_{x}^{s m}$ and the shape matrix $R_{x}^{s m}$ of the statebounding zonotope $\hat{\mathcal{X}}^{s m}$ corrected by the $i^{\text {th }}$ output, i.e.,

$$
\hat{\mathcal{X}}_{i}^{s m}=\left\langle c_{x}^{s m}, R_{x}^{s m}\right\rangle,
$$

can be computed by intersecting the prediction state set $\mathcal{P}^{s m}=\left\langle c_{p}^{s m}, R_{p}^{s m}\right\rangle$, where $c_{p}^{s m}$ and $R_{p}^{s m}$ denote the center and shape matrix of the zonotope $\mathcal{P}_{k}^{s m}$, respectively, and the set of states consistent with each output strip $\mathcal{S}_{y_{i}}$, yielding

$$
\begin{aligned}
c_{x}^{s m} & =c_{p}^{s m}+\lambda_{i}\left(y_{i}-C_{i} c_{p}^{s m}\right), \\
R_{x}^{s m} & =\left[\begin{array}{ll}
\left(I-\lambda_{i} C_{i}\right) R_{p}^{s m} & -\lambda_{i} E_{v_{i}}
\end{array}\right],
\end{aligned}
$$

with

$$
\begin{aligned}
c_{p}^{s m} & =A c_{x,-}^{s m}+B u_{-}, \\
R_{p}^{s m} & =\left[\begin{array}{ll}
A R_{x,-}^{s m} & E_{\omega}
\end{array}\right],
\end{aligned}
$$

where $\lambda$ is a vector that provides degrees of freedom to tune the system monitoring, e.g., optimizing the state-bounding 
zonotope to be as robust as possible with respect to the effect of uncertainties.

Proof: Considering the dynamical model (4), the prediction state set can be computed as a zonotope, i.e.,

$$
\mathcal{P}^{s m}=\left\langle c_{p}^{s m}, R_{p}^{s m}\right\rangle .
$$

Furthermore, $c_{p}^{s m}$ and $R_{p}^{s m}$ can be calculated using (10) at time instant $k$. Additionally, a strip $\mathcal{S}_{y}$ is computed by considering each measurement component $y$ as

$$
\mathcal{S}_{y_{i}}=\left\{x \in \mathbb{R}^{n_{x}}:\left|C_{i} x-y_{i}\right| \leq E_{v_{i}}\right\} .
$$

According to [4], the intersection between the zonotope in (11) and the obtained strip in (12) provides the state estimation using SMA. Therefore, the time evolution of the center and the segments of the state-bounding zonotope (8) are given as it is derived in (9).

Remark 3.1: According to [16], in order to apply the SMA to the multi-output case, i.e., $n_{y}>1$, the system can be considered as several single-output systems based on the dimension of the outputs $n_{y}$. Considering the system as several single-output systems lead to compute vectors $\lambda_{1}, \lambda_{2}, \ldots, \lambda_{n_{y}}$, independently.

An optimal tuning of the parameter $\lambda$ will be addressed next.

\section{Comparative assessment}

The state estimation using zonotopic IOA and SMA is introduced in Sections III-A and III-B, respectively. The main goal of this section is to compare both approaches from the state estimation point of view. Just for sake of clarity, the analytical comparison will be focused on the singlemeasurement case in this section as in [4]. But, according to [16], the generalization of the comparison can be easily extended to the multi-measurement case considering Remark 3.1. It is worth mentioning that there are other manners to formulate the state estimation for the multi-measurement case using SMA based on [16].

So far, comparing (6) with (9), it is still not evident how to relate both state estimation approaches because of the different temporal information of the measured output used for each approach. More precisely, the state estimation using IOA considers information of the output measurements at the previous time instant while state estimation using SMA is obtained by using the information of the measurements at the current time instant. Moreover, another difference that can be observed from the comparison of (6) with (9) is related to the term that provides degrees of freedom to tune the system monitoring. In the case of IOA, the tuning is done by means of the observer gain $L^{i o}$. On the other hand, the same role can be achieved by means of the parameter $\lambda$ in the case of SMA. Therefore, the relationship of IOA and SMA depends on the satisfaction of the following points: i) Synchronizing the structure of the state estimation approaches such that both use the measured output $y$ given at the same time instant. ii) Finding the condition to relate the observer gain $L^{i o}$ and parameter $\lambda$
In this regard, a discussion of such relationship considering the mentioned points will be the main topic of the next section.

\section{ON THE RELATIONSHIP OF THE ZONOTOPIC IOA AND SMA}

\section{A. Output synchronization}

As it was mentioned in Section III-C, the first point that should be considered in order to relate IOA and SMA is related to the fact that both state estimation approaches should be synchronized such that both use the measured output $y$ at the same time instant. In this regard and following [17], the IOA proposed by [14] can be modified leading to a zonotopic current IOA (CIOA) given by

$$
\hat{\mathcal{X}}^{\text {cio }}=\left\langle c_{x}^{\text {cio }}, R_{x}^{c i o}\right\rangle
$$

where $c_{x}^{c i o}$ and $R_{x}^{c i o}$ denote the center and the shape matrix of the zonotope (13) bounding the set of estimated states. Following the same idea as Proposition 3.1, by introducing a priori estimated set in prediction step as $\mathcal{P}^{\text {cio }}=\left\langle c_{p}^{c i o}, R_{p}^{\text {cio }}\right\rangle$, both center and shape matrix of $\hat{\mathcal{X}}^{\text {cio }}$ can be propagated as

$$
\begin{aligned}
& c_{x}^{c i o}=c_{p}^{c i o}+L^{c i o}\left(y-C c_{p}^{c i o}\right), \\
& R_{x}^{c i o}=\left[\begin{array}{ll}
\left(I-L^{c i o} C\right) \bar{R}_{p}^{c i o} & -L^{c i o} E_{v}
\end{array}\right] \text {, }
\end{aligned}
$$

with

$$
\begin{aligned}
c_{p}^{c i o} & =A c_{x,-}^{c i o}+B u_{-}, \\
R_{p}^{c i o} & =\left[\begin{array}{ll}
A \bar{R}_{x,-}^{c i o} & E_{\omega}
\end{array}\right] .
\end{aligned}
$$

As it can be seen from (14), the state estimation set $\hat{\mathcal{X}}^{\text {cio }}$ can be performed based on the information of the measurement given at the current time instant. When using CIOA, the similarity of the $c_{p}^{c i o}$ with the $c_{p}^{s m}$ and also $R_{p}^{\text {cio }}$ with $R_{p}^{s m}$ can be observed by comparing (14) with (9). It can also be noted that, using the same initial condition for both approaches, i.e., $x_{0}$ belongs to the both initial zonotope $\hat{\mathcal{X}}_{0}^{\text {cio }}=\left\langle c_{x, 0}^{\text {cio }}, R_{x, 0}^{\text {cio }}\right\rangle$ and $\hat{\mathcal{X}}_{0}^{s m}=\left\langle c_{x, 0}^{s m}, R_{x, 0}^{s m}\right\rangle$, and taking into account the formulation of CIOA, the only difference between both approaches is related to the different manners of selecting the observer gain $L^{\text {cio }}$ and the parameter $\lambda$. Generally speaking, in the case that $\lambda$ in SMA and $L^{\text {cio }}$ in IOA are identical, the same state-bounding zonotopes will be obtained.

\section{B. Design the observer gain and parameter $\lambda$}

As it is mentioned before, both $L^{\text {cio }}$ and $\lambda$ play the same role to tune the system monitoring. Considering the zonotopic-based method, the size of the zonotope is mainly influenced by the uncertainties. When designing either $L^{\text {cio }}$ or $\lambda$ for state-observation purposes, the main goal will be only reducing the effect of the state estimation uncertainty.

As it was mentioned in Section IV-A, in order to synchronize IOA and SMA to have the same state estimation structure, CIOA can be used. Then, the optimal observer gain for the case of CIOA is obtained by following Theorem 4.1. 
Theorem 4.1: (Optimal observer gain) Considering the structure of CIOA in (14) and introducing the tuning parameters $Q=Q^{\top} \geq 0$ and $R=R^{\top}>0$, if there exist positive scalars $\gamma$, the optimal observer gain $L^{c i o, *}$ is computed as

$$
L^{c i o, *}=\Upsilon^{-1} W
$$

where $\Upsilon$ and $W$ are the feasible solutions of the following optimization problem:

$$
\min \gamma
$$

subject to the following LMIs:

$$
\left[\begin{array}{cc}
\gamma I_{n_{x}} & I_{n_{x}} \\
I_{n_{x}} & \Upsilon
\end{array}\right]>0
$$

$$
\left[\begin{array}{ccc}
\Upsilon A+A^{\top} \Upsilon-W C-C^{\top} W^{\top}+\Upsilon 2 \eta & \Upsilon\left(Q^{\frac{1}{2}}\right) & W \\
Q^{\frac{1}{2}} \Upsilon & -I & 0 \\
W^{\top} & 0 & -R^{-1}
\end{array}\right]<0
$$

where $\eta>0$ is the decay rate.

Proof: According to [14], minimizing the F-radius of a zonotope is equivalent to minimize the trace of its covariance. In this regard, the F-radius of (14) is obtained as

$$
J^{\text {cio }}=\left\|R_{x}^{\text {cio }}\right\|_{F}^{2}=\operatorname{tr}\left(R_{x}^{\text {cio }} R_{x}^{\text {cio }}{ }^{\top}\right),
$$

where $J^{\text {cio }}$ denotes the Frobenius radius of the zonotope matrix $R_{x}^{\text {cio }}$. According to [18], a possible approach to minimize the obtained criterion (18) is to compute the observer gain $L^{\text {cio }}$ that guarantees $J^{\text {cio }}$ is lower than some given number $\gamma$. Moreover, introducing the Lyapunov function

$$
V(x)=x^{\top} P x,
$$

where $P>0 \in \mathbb{R}^{n_{x} \times n_{x}}$, such that the following conditions are satisfied:

$$
\begin{aligned}
& V\left(x_{0}\right)=x_{0}^{\top} P x_{0}<\gamma, \\
& V\left(x_{+}\right)-V(x)+J^{c i o}<0 .
\end{aligned}
$$

By introducing $\Upsilon=P^{-1}$, the condition in (20a) can be rewritten as

$$
\gamma I_{n_{x}}-\Upsilon^{-1}>0
$$

Now, applying the Schur complement, (21) can be reshaped into the LMI form as (17b). Moreover, by recalling $V\left(x_{+}\right)=x_{+}^{\top} P x+x^{\top} P x_{+}$and considering (4) and (5), (20b) can be rewritten as

$$
\left(A-L^{\text {cio }} C\right) P+P\left(A-L^{\text {cio }} C\right)^{\top}+Q+L^{\text {cio }} R\left(L^{\text {cio }}\right)^{\top}<0 .
$$

Now, by introducing the decay term rate $\eta$ in order to ensure the fast dynamic response of the observer, (22) can be rewritten as

$\left(A-L^{\text {cio } C}\right) P+P\left(A-L^{\text {cio }} C\right)^{\top}+2 \eta P+Q+L^{\text {cio }} R\left(L^{\text {cio }}\right)^{\top}<0$.

Then, left and right multiplications of (22) by $\Upsilon=P^{-1}$ and introducing $W=\Upsilon L^{c i o, *},(23)$ becomes

$$
\Upsilon A-W C+A^{\top}-C^{\top} W^{\top}+\Upsilon 2 \eta+\Upsilon Q \Upsilon+W R W^{\top}<0 .
$$

Now, by reformulating (24) as

$$
\begin{aligned}
& \Upsilon A+A^{\top} \Upsilon-W C-C^{\top} W^{\top}+\Upsilon 2 \eta \\
& -\left[\Upsilon\left(Q^{\frac{1}{2}}\right) \quad W\right]\left[\begin{array}{cc}
-I & 0 \\
0 & -R
\end{array}\right]\left[\begin{array}{c}
Q^{\frac{1}{2}} \Upsilon \\
W^{\top}
\end{array}\right]<0 .
\end{aligned}
$$

Again, by applying the Schur complement, (25) is reshaped into the LMI form as (17c). Hence, if it is desired to minimize the value of $\gamma$, the optimization problem (17) should be solved. Finally, the observer gain is thus obtained as (16).

Remark 4.1: It is worth mentioning that, according to [19], $L^{c i o, *}$ can also be computed in the classical manner using the weighted Frobenius norm $\left(F_{W^{-}}\right.$ radius) of the state bounding zonotope $\hat{\mathcal{X}}^{\text {cio }}$ as $L^{c i o, *}=\left(R_{x}^{c i o} R_{x}^{c i o \top} C^{\top}\right)\left(C R_{x}^{c i o} R_{x}^{c i o \top} C^{\top}+E_{v} E_{v}^{\top}\right)^{-1}$. But, the benefit of using the proposed approach is the ability of using it in the nonlinear case.

On the other hand, considering SMA, $\lambda$ should be designed with the aim of reducing the effect of the state estimation uncertainties. In this regard, the optimal value of $\lambda$ (denoted as $\lambda^{*}$ ) in the case of SMA can be computed by means of Theorem 4.2.

Theorem 4.2: (Optimal $\lambda$ ) Considering the structure of SMA in (9) and introducing the tuning parameters $Q=$ $Q^{\top} \geq 0$ and $R=R^{\top}>0$, if there exist positive scalars $\gamma, \lambda^{*}$ can be obtained as

$$
\lambda^{*}=\Upsilon^{-1} W,
$$

where $\Upsilon$ and $W$ are obtained by solving the following optimization problem:

$$
\min \gamma,
$$

subject to the following LMIs:

$$
\left[\begin{array}{cc}
\gamma I_{n_{x}} & I_{n_{x}} \\
I_{n_{x}} & \Upsilon
\end{array}\right]>0
$$

$$
\left[\begin{array}{ccc}
\Upsilon A+A^{\top} \Upsilon-W C-C^{\top} W^{\top}+\Upsilon 2 \eta & \Upsilon\left(Q^{\frac{1}{2}}\right) & W \\
Q^{\frac{1}{2}} \Upsilon & -I & 0 \\
W^{\top} & 0 & -R^{-1}
\end{array}\right]<0,
$$

where $\eta>0$ is the decay rate.

Proof: The proof follows from the same procedure of results presented in Theorem 4.1 to the SMA case.

Remark 4.2: It is worth mentioning that, according to [4], $\lambda^{*}$ can also be computed in the classical manner using the weighted Frobenius norm $\left(F_{W^{-}}\right.$ radius) of the state bounding zonotope $\hat{\mathcal{X}}^{s m}$ as $\lambda_{i}=$ $\left(R_{x}^{s m} R_{x}^{s m^{\top}} C_{i}^{\top}\right)\left(C_{i} R_{x}^{s m} R_{x}^{s m^{\top}} C_{i}^{\top}+E_{v_{i}} E_{v_{i}}^{\top}\right)^{-1}$. But, the benefit of using the proposed approach is the ability of using it in the nonlinear case.

\section{Discussion}

The main difference between SMA and IOA is related to the different way that measurements are taken into account to obtain the state estimation. In the case of IOA, the state estimation is obtained in an explicit way by combining the model and the measurements through the observer gain. On 


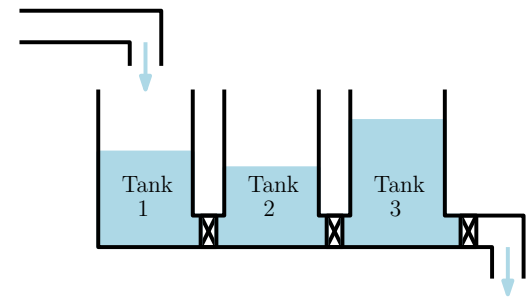

Fig. 1: Schematic diagram of the three-tank system.

the other hand, SMA carries out this process implicitly by means of the intersection between the set of states consistent with the model and the measurements. More precisely, the state estimation using IOA considers information of the output measurements at the previous time instant while state estimation using SMA is obtained by using the information of the measurements at the current time instant. In this regard, both state estimation approaches should be synchronized such that both use the measured output $y$ at the same time instant. The synchronization of the output $y$ is performed using CIOA as explained in Section IV-A. After that, by having the same structure for the both state estimation approaches, the only difference is related to the different manners of selecting the observer gain $L^{\text {cio }}$ and the parameter $\lambda$. Therefore, using the zonotopic structure of CIOA in (14) and the zonotopic structure of SMA in (9), the observer gain $L^{\text {cio }}$ and the parameter $\lambda$ are designed based on LMI techniques, the results are reported in Theorems 4.1 and 4.2. The similarity of the LMIs in (17) for computing the optimal observer gain $L^{c i o, *}$ in the case of CIOA and the LMIs in (27) for computing the optimal parameter $\lambda^{*}$ in the case of SMA can be observed. Thus, the same state estimation can be obtained considering the points mentioned in Sections IV-A and IV-B.

\section{CAse Study}

\section{A. System description}

The comparative analysis of IOA and SMA will be performed by using a three-tank system. The scheme of the considered case study is shown in Figure 1. The three-tank system, as represented in Figure 1, consists of three identical tanks that are connected by using pipes. The first tank has an incoming flow that can be controlled by means of a pump (actuator) and the system output (outflow) is located in the last tank. Moreover, the state vectors contains the water levels of the tanks.

Since all the mathematical developments in previous sections were obtained based on the consideration of a linear dynamic system, the linearized model of the three-tank system is required to be obtained to illustrate the effectiveness of the characterized formulations ${ }^{1}$. Therefore, considering the following working point:

$$
\text { - } h_{1}^{*}=0.51 \mathrm{~cm}, \quad h_{2}^{*}=0.50 \mathrm{~cm}, \quad h_{3}^{*}=0.35 \mathrm{~cm} \text {, }
$$

\footnotetext{
${ }^{1}$ The description of the nonlinear mathematical model and it linearization process are suppressed because of the space limitation, but it can be obtained based on mass balance and Bernoulli's law.
}

TABLE I: $L^{c i o, *}$ and $\lambda^{*}$ in steady state.

\begin{tabular}{ccc}
\hline \multicolumn{5}{c}{ Classical approach } \\
\\
$L^{\text {cio,* }}$ \\
\hline$\left[\begin{array}{lll}0.7389 & 0.0070 & 0.0608 \\
0.0293 & 0.7504 & 0.0265 \\
0.0619 & 0.0250 & 0.7914\end{array}\right] \quad\left[\begin{array}{lll}0.7389 & 0.0070 & 0.0608 \\
0.0293 & 0.7504 & 0.0265 \\
0.0619 & 0.0250 & 0.7914\end{array}\right]$ \\
\hline
\end{tabular}

\begin{tabular}{|c|c|c|c|c|c|}
\hline \multicolumn{6}{|c|}{ Proposed approach } \\
\hline & $L^{c i o, *}$ & & & $\lambda^{*}$ & \\
\hline 0.7268 & 0.0063 & 0.06127 & 0.7268 & 0.0063 & 0.0612 \\
\hline 0.0223 & 0.7365 & 0.0236 & 0.0223 & 0.7365 & 0.0236 \\
\hline 0.0602 & 0.0235 & 0.7614 & 0.0602 & 0.0235 & 0.7614 \\
\hline
\end{tabular}

and using the Euler discretization with a sampling time of $1 \mathrm{~s}$, the linearized model can be stated in the state-space form as

$$
\begin{aligned}
h_{k+1} & =A h_{k}+B u_{k}+E_{\omega} \omega_{k}, \\
y_{k} & =C h_{k}+E_{v} v_{k}
\end{aligned}
$$

where

$$
\begin{aligned}
A & =\left[\begin{array}{ccc}
0.9712 & 0 & 0 \\
0.0288 & 0.9638 & 0.0074 \\
0 & 0.0074 & 0.9877
\end{array}\right], \quad B=\left[\begin{array}{c}
64.9351 \\
0 \\
0
\end{array}\right], \\
C & =\left[\begin{array}{lll}
0 & 0 & 1
\end{array}\right] .
\end{aligned}
$$

Moreover, $\omega$ and $v$, influencing all the statespace directions and the output, are modeled with $E_{\omega}=\operatorname{diag}\left(\begin{array}{lll}0.05 & 0.05 & 0.05\end{array}\right)$ and $E_{v}=0.08$, respectively.

\section{B. State estimation}

The main goal of this section is to compare the state estimation using IOA and SMA. Regarding the computation of $L^{c i o, *}$ and $\lambda^{*}$, in one hand, they can be computed in a classical manner according to [19] and [4], respectively. On the other hand, the proposed Theorems 4.1 and 4.2 can be utilized to compute $L^{\text {cio,* }}$ and $\lambda^{*}$, respectively. The obtained results using both classical and proposed approaches can be seen in Table I.

As can be seen from Table I, the obtained results using the classical manner proposed by [19] and the proposed approaches in Theorems 4.1 and 4.2 are almost the same. Furthermore, comparing the classical manner and the proposed approach for computing the $L^{c i o, *}$ and $\lambda^{*}$, separately, reveals the equivalence of the CIOA and SMA in state estimation framework. Moreover, considering the the steadystate operation of the mentioned three-tank system, the state estimation zonotopes computed by CIOA and SMA considering the proposed approach in Theorems 4.1 and 4.2 for computing the $L^{c i o, *}$ and $\lambda^{*}$ can be seen in Figures 2 and 3, respectively. Furthermore, the time evaluations of the interval hulls of the state estimation using both CIOA and SMA are projected into the output space by using the system matrix $C$. Figure 4 is obtained from the simulation as a result of the projection of the state estimation into the output space. 


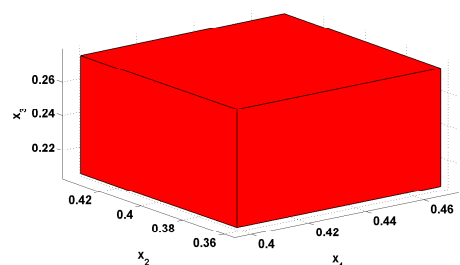

Fig. 2: State estimation using CIOA in steady state using the proposed approach.

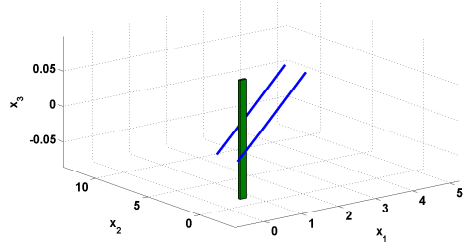

(a) Intersection the the prediction state set and the set of states consistent with each output strip.

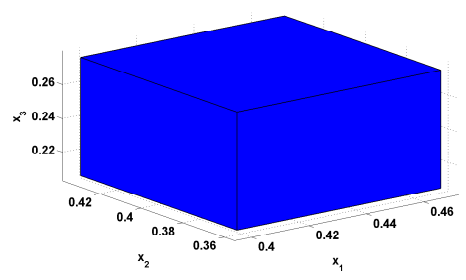

(b) Obtained state set after intersection

Fig. 3: State estimation using SMA in steady state using the proposed approach.

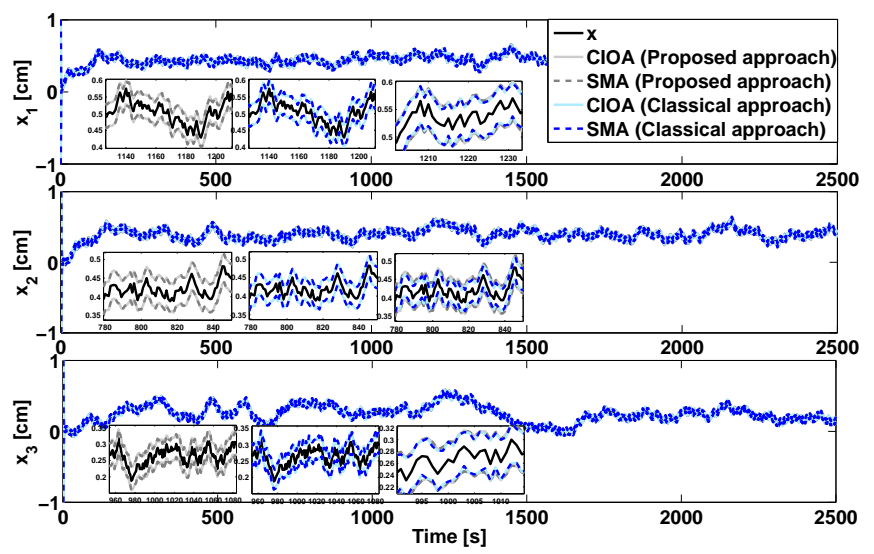

Fig. 4: Projection of the state estimations into the output space.

As can be seen from (4), maximum and minimum bounds of the obtained zonotopic state estimations using both CIOA and SMA are identical. This fact illustrates that the proposed observer design is well suited to relate CIOA and SMA.

\section{CONCLUSIONS}

This paper has proposed a robust zonotopic observer design for discrete-time linear systems. First, state estima- tion using both interval observer approach (IOA) and setmembership approach (SMA) is analyzed, separately. Then, the comparison and the condition of relating both approaches are proposed using the current interval observer approach (CIOA) and the Linear Matrix Inequality (LMI) technique. The effect of the state disturbance and measurement noise are taken into account using a zonotopic representation of the involved sets. Finally, a three-tank system is considered as a case study for illustrating the effectiveness of the proposed observer design.

\section{ACKNOWLEDGEMENT}

This work has been partially funded by the Spanish Government (MINECO) through the project DEOCS (ref. DPI2016-76493-C3-3-R), by MINECO and FEDER through the project HARCRICS (ref. DPI2014-58104-R) and by Agència de Gestió d'Ajuts Universitaris i de Recerca.

\section{REFERENCES}

[1] M. Blanke, M. Kinnaert, J. Lunze, M. Staroswiecki, J. Schröder, Diagnosis and fault-tolerant control, Vol. 691, Springer, 2006.

[2] J. Gertler, Fault detection and diagnosis in engineering systems, CRC Press, USA, 1998.

[3] V. Puig, S. Montes de Oca, J. Blesa, Adaptive threshold generation in robust fault detection using interval models: time-domain and frequency-domain approaches, International Journal of Adaptive Control and Signal Processing 27 (10) (2013) 873-901.

[4] T. Alamo, J. M. Bravo, E. F. Camacho, Guaranteed state estimation by zonotopes, Automatica 41 (6) (2005) 1035-1043.

[5] T. Raïssi, D. Efimov, A. Zolghadri, Interval state estimation for a class of nonlinear systems, IEEE Transactions on Automatic Control 57 (1) (2012) 260-265.

[6] R. E. Kalman, A new approach to linear filtering and prediction problems, Journal of Basic Engineering 82 (1) (1960) 35-45.

[7] P. S. Maybeck, R. D. Stevens, Reconfigurable flight control via multiple model adaptive control methods, IEEE Transactions on Aerospace and Electronic systems 27 (3) (1991) 470-480.

[8] F. Schweppe, Recursive state estimation: Unknown but bounded errors and system inputs, IEEE Transactions on Automatic Control 13 (1) (1968) 22-28.

[9] J. Meseguer, V. Puig, T. Escobet, J. Saludes, Observer gain effect in linear interval observer-based fault detection, Journal of Process Control 20 (8) (2010) 944-956.

[10] G. M. Ziegler, Lectures on polytopes (graduate texts in mathematics), Springer, USA, 1994.

[11] W. Kühn, Rigorously computed orbits of dynamical systems without the wrapping effect, Computing 61 (1) (1998) 47-67.

[12] D. Efimov, T. Raïssi, S. Chebotarev, A. Zolghadri, Interval state observer for nonlinear time varying systems, Automatica 49 (1) (2013) 200-205.

[13] F. Mazenc, O. Bernard, Interval observers for linear time-invariant systems with disturbances, Automatica 47 (1) (2011) 140-147.

[14] C. Combastel, Zonotopes and Kalman observers: Gain optimality under distinct uncertainty paradigms and robust convergence, Automatica 55 (2015) 265-273.

[15] F. Xu, V. Puig, C. Ocampo-Martinez, S. Olaru, F. Stoican, Set-theoretic methods in robust detection and isolation of sensor faults, International Journal of Systems Science 46 (13) (2015) 2317-2334.

[16] V. T. H. Le, C. Stoica, T. Alamo, E. F. Camacho, D. Dumur, Zonotopes: From guaranteed state-estimation to control, John Wiley \& Sons, 2013.

[17] K. Ogata, Discrete-time control systems, Englewood Cliffs, N.J., Prentice-Hall, USA, 1995.

[18] G. Duc, Introduction aux LMI. lecture notes. Supélec (2002).

[19] M. Pourasghar, V. Puig, C. Ocampo-Martinez, Interval observer versus set-membership approaches for fault detection in uncertain systems using zonotopes, International Journal of Robust and Nonlinear Control 29 (10) (2019) 2819-2843. 